\title{
The vicious and virtuous circles of clonal hematopoiesis
}

\author{
Clonal hematopoiesis can exist as both a driver and a consequence of inflammatory dysregulation.
}

\author{
Nili Furer, Nathali Kaushansky and Liran I. Shlush
}

T he expansion of hematopoietic stem/progenitor cells (HSPCs) with specific, recurrent genetic variants in people without a diagnosis of hematological malignancy is called 'clonal hematopoiesis' $(\mathrm{CH})^{1}$. $\mathrm{CH}$ is composed of many diverse entities and can be subcategorized according to the specific gene involved, the mutation subtype, the etiology, and related clinical features, including age, blood-count effects and associated comorbidities. The mutational subtypes of $\mathrm{CH}$ are defined by the presence of single-nucleotide variants and small insertions and/or deletions, or large-scale mosaic chromosomal alterations (mCAs), and its etiology can be infectious, genotoxic, autoimmune or metabolic, with each providing distinct fitness advantages to clones. Two articles published in this issue of Nature Medicine link novel etiological and context-specific clinical features to $\mathrm{CH}$. Dharan et al. present chronic infection with human immunodeficiency virus (HIV) as a novel etiology for $\mathrm{CH}$, characterized by specific gene associations and clonal dynamics ${ }^{2}$, whereas Zekavat et al. demonstrate the association of mCAs with a wide range of infections, including infection with the coronavirus SARS-CoV- $2^{3}$. Key to the connection between these manuscripts is the self-perpetuating circle of inflammation and clonal expansion.

Zekavat et al. studied the association between mCAs and risk of infection among 768,762 people $^{3}$. They found expanded autosomal mCAs to be significantly associated with diverse infections, including respiratory, gastrointestinal and genitourinary infections, as well as with elevated lymphocyte counts. The authors suggest that mCAs could confer an increased predisposition to infection, and that surveillance for mCAs could identify people at high risk. Of note, this study and others have found a strong correlation between mCAs and increased lymphocyte count, as well as chronic lymphocytic leukemia ${ }^{4}$, a condition that predisposes people to infection through dysregulated

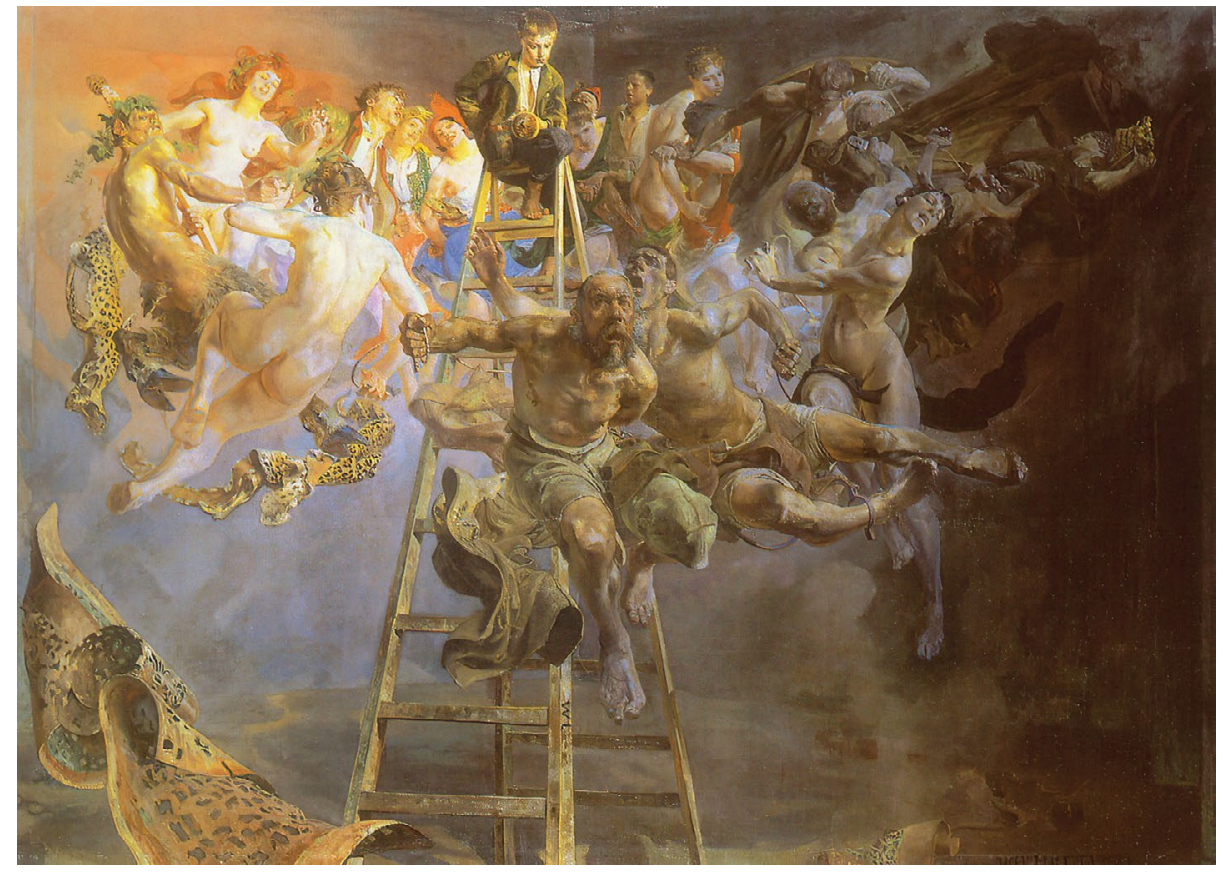

Vicious Circle by Jacek Malczewski. Credit: Artifact / Alamy Stock Photo

lymphocyte differentiation and function. This correlation strongly implicates some form of immunological aberration as the basis for the association of these conditions with infection. In addition, these findings highlight the need to distinguish mCAs associated with monoclonal B cell expansion (as in monoclonal B cell lymphocytosis or chronic lymphocytic leukemia) from those originating in HSPCs, as well as the potentially different consequences of each. Further studies simultaneously analyzing mCAs and single-nucleotide variants or insertions and/or deletions are also called for in order to clarify the association between ' $\mathrm{CH}$-associated mCAs' and infection.

Dharan et al. studied the prevalence of $\mathrm{CH}$ in $\mathrm{HIV}$-positive people ${ }^{2}$, in view of observations that infection with HIV, like $\mathrm{CH}$, is linked to certain age-related specific comorbidities (cardiovascular disease and
non-AIDS-defining malignancies). The authors recruited $220 \mathrm{HIV}$-positive people and $226 \mathrm{HIV}$-negative control participants, $>55$ years of age, and compared these groups on the basis of the prevalence and type of somatic mutations. In accordance with previous findings, the most common genes mutated were DNMT3A and TET2 $2^{5}$. CH was more prevalent in HIV-positive participants than in HIV-negative participants across all age groups. No significant differences between the two groups were noted in median variant allele fractions (VAFs), the number of $\mathrm{CH}$ mutations per person, or specific genes involved; nevertheless, mean VAFs of identified mutations increased with age at a greater rate in the HIV-positive cohort. Interestingly, $\mathrm{CH}$ and infection with HIV were independently associated with increases in blood parameters associated with chronic inflammation, including neutrophil 
count and increased levels of the cytokine IL-6 and C-reactive protein in serum.

A growing body of evidence suggests that factors other than aging can result in a predisposition to $\mathrm{CH}$ and may be responsible for the wide variability in clonal dynamics across $\mathrm{CH}$-positive people. Among genetically predisposing factors, the most notable involve the TERT loci and TET2 variants ${ }^{6}$. Toxic exposures such as chemotherapy or radiotherapy are highly specific for mutations in PPM1D, TP53 and CHEK2, and smoking is specifically associated with mutations in ASXL1 ${ }^{7}$. Inflammation has been shown to strongly affect TET2 clonal dynamics via increased IL-6 production ${ }^{8}$ and to strongly affect DNMT3A clones via an immune response mediated by the cytokine IFN- $\gamma^{9}$. Immune attack, as occurs in aplastic anemia, is associated with mutations in $B C O R$ and $B C O R L 1$, suggestive of a role of autoimmunity in clonal selection ${ }^{10}$. Metabolic factors also contribute to a predisposition to $\mathrm{CH}$, as demonstrated by the association of hyperglycemia and insulin resistance with mutations in $T E T 2^{11}$, and atherosclerosis has been shown to promote HSPC division and accelerate clonal evolution ${ }^{12}$.

Common to most of the factors mentioned above is some form of chronic inflammatory dysregulation, which can be age related and of infectious, autoimmune or metabolic origin. Chronic inflammation has been shown to exhaust the native HSPC pool as a result of excessive differentiation, depleting its self-renewal capacity. $\mathrm{CH}$-mutant HSPCs, on the other hand, demonstrate defective differentiation and enhanced self-renewal under the influence of certain pro-inflammatory cytokines and chemokines. Thus, repeated or sustained exposure to inflammatory stimuli might leave them at a competitive advantage. The studies presented in this issue $e^{2,3}$ describe the role of $\mathrm{CH}$ as both a driver of inflammation (primary $\mathrm{CH}$ ) and its consequence (secondary $\mathrm{CH}$ ). This could be conceptualized chronologically as follows: low-grade inflammation initially promotes clonal expansion of certain mutant HSPCs that, as the clone size increases, further promotes inflammation in a vicious circle that ultimately compromises organ function.

Of note, $\mathrm{CH}$ can also be virtuous in nature. Studies have demonstrated its potentially beneficial effects in the context of bone marrow transplantation, through its association with chronic graft-versus-host disease and decreased risk of relapse ${ }^{13}$. Patients with aplastic anemia frequently develop $\mathrm{CH}$ characterized by mutations in BCOR and BCORL1; these patients' favorable outcome suggests that these mutations confer some fitness advantage to the clone, allowing it to evade immune attack by autoreactive T cells ${ }^{10}$. These adaptation mechanisms of the marrow, beneficial to the organism's fitness at some early point in time, yet carrying detrimental consequences on its fitness later on, are reminiscent of the 'antagonistic pleiotropy' theory of aging. Despite vast improvements in the understanding of $\mathrm{CH}$, important questions remain unanswered. Further work is needed for full elucidation of the role of $\mathrm{CH}$ as both product and perpetrator of inflammation, as well as the specific contexts in which these may be harmful or beneficial.

\section{Nili Furer, Nathali Kaushansky and} Liran I. Shlush (D)

Department of Immunology, The Weizmann Institute of Science, Rehovot, Israel.

$\bigotimes_{e-m a i l: l i r a n s h l u s h 3 @ g m a i l . c o m}$

Published online: 7 June 2021

https://doi.org/10.1038/s41591-021-01396-5

References

1. Shlush, L. I. Blood 131, 496-504 (2018).

2. Dharan, N. J. Nat. Med. https://doi.org/10.1038/s41591-02101357-y (2021).

3. Zekavat, S. M. Nat. Med. https://doi.org/10.1038/s41591-02101371-0 (2021).

4. Laurie, C. C. et al. Nat. Genet. 44, 642-650 (2012).

5. Buscarlet, M. et al. Blood 130, 753-762 (2017).

6. Bick, A. G. et al. Nature 586, 763-768 (2020).

7. Bolton, K. L. et al. Nat. Genet. 52, 1219-1226 (2020).

8. Meisel, M. et al. Nature 557, 580-584 (2018)

9. Hormaechea-Agulla, D. et al. Cell Stem Cell https://doi. org/10.1016/j.stem.2021.03.002 (2021).

10. Yoshizato, T. et al. N. Engl. J. Med. 373, 35-47 (2015).

11. Cai, Z. et al. J. Clin. Invest. 131, e140707 (2021).

12. Heyde, A. et al. Cell 184, 1348-1361.e1322 (2021).

13. Frick, M. et al. J. Clin. Oncol. 37, 375-385 (2019).

Competing interests

The authors declare no competing interests.

\title{
Putting the MD back into MDMA
}

\author{
A phase 3 study shows that MDMA may be a promising treatment for PTSD, which will require a shift in how this \\ drug is perceived.
}

\section{David J. Nutt and Harriet de Wit}

$\mathrm{M}$ DMA-colloquially known in its unregulated form as 'E' or 'ecstasy' in Europe and as 'molly' in the USA-is a small, amphetamine-like molecule that has had a rollercoaster reputational ride, from being positioned as a promising new therapeutic tool to being branded a brain-damaging recreational drug. Most of those historic fears were overstated, and recent empirical research, especially into the treatment of post-traumatic stress disorder (PTSD) and related conditions, is now bringing MDMA back into the medical fold. In this issue of Nature Medicine, Mitchell et al. report the first phase 3 study of MDMA, which reveals significant efficacy and an excellent safety profile in people with severe $\mathrm{PTSD}^{1}$. It now seems likely that it will be an approved medication in a few years.

MDMA was invented by Merck in 1912 as a precursor in a new synthesis for hemostatic substances ${ }^{2}$; Merck tested MDMA in animal models in 1927 and in 1959 but found nothing of interest. It was then resurrected by Alexander Shulgin in the 1970s, when he self-experimented with a range of phenylethylamine drugs. MDMA stood out as being different from other related compounds, as it provided greater clarity of thought and empathy with others. Shulgin introduced it to his wife, who was a psychotherapist, and she agreed it had therapeutic potential, particularly for couples counseling. The Shulgins made it available to other therapists in the USA, who found MDMA helpful in breaking down the hostile tensions and frictions that build up in many partnerships. For these 